\title{
Two Conjectures of B. R. Santos Concerning Totitives
}

\author{
By H. G. Kopetzky and W. Schwarz
}

Abstract. Recently B. R. Santos conjectured that 12 is the largest integer $n$ with the following property:

(*)

$$
\left\{\begin{array}{l}
\text { If } m \in[1, n] \text { and } n \text { are relatively prime, then } \\
n+m \text { is a prime number. }
\end{array}\right.
$$

Using deep numerical estimates of Rosser and Schoenfeld for the number $\pi(x)$ of primes less than $x$, it is proved that the conjecture of Santos is true. The same result holds, if in addition it is assumed in (*) that $m$ is a prime.

The positive integers not greater than a given integer and coprime to it are called its totitives. It is well known that 30 is the largest integer with the property that all its totitives are prime.

B. R. Santos [4] proved that there exists a largest integer $n$ with the property

$$
1 \leqslant m \leqslant n, \text { g.c.d. }(m, n)=1 \Rightarrow(n+m \text { is prime }) \text {. }
$$

He conjectured that $n=12$ is the largest integer having the property $\left(P_{1}\right)$. Furthermore, he conjectures that there is a largest integer $n$ with the property

$\left(\mathrm{P}_{2}\right) \quad 1 \leqslant m \leqslant n$, g.c.d. $(m, n)=1$ and $m$ prime $\Rightarrow(n+m$ is prime $)$.

In this note we prove the following results.

(A) $n=12$ is the largest integer with the property $\left(\mathrm{P}_{1}\right)$.

(B) $n=12$ is the largest integer with the property $\left(\mathrm{P}_{2}\right)$.

Denote by $\pi(x)$ the number of primes not greater than $x$, and by $\varphi(n)$ Euler's function.

(A) is true if

$$
\varphi(n)>\pi(2 n)-\pi(n)
$$

holds for $n>12$. In order to prove (1) we use the following estimates due to Rosser and Schoenfeld [2, Theorems 1 and 15].

$$
\begin{array}{ll}
\pi(x)>f(x):=\frac{x}{\log x}\left(1+\frac{1}{2 \log x}\right) & \text { for } x \geqslant 59 \\
\pi(x)<g(x):=\frac{x}{\log x}\left(1+\frac{3}{2 \log x}\right) & \text { for } x>1,
\end{array}
$$

Received May 18, 1978.

AMS (MOS) subject classifications (1970). Primary 10A25; Secondary 10H15, 10-04.

Key words and phrases. Prime number theorem, prime totitives. 
and *

(RS 3)

$$
\frac{n}{\varphi(n)}<e^{c} \log \log n+\frac{5}{2 \log \log n} \text { for } n \geqslant 3,
$$

except when $n=n_{0}=2 \cdot 3 \cdot 5 \cdot 7 \cdot 11 \cdot 13 \cdot 19 \cdot 23$. For this special $n$ the constant $5 / 2$ in (RS 3) has to be replaced by 2.50637 .

Define for $x \geqslant 3$ the function $h(x)$ by

$$
h(x)=x \cdot\left(e^{c} \log \log x+\frac{5}{2 \log \log x}\right)^{-1} .
$$

A simple computation shows that the function $F(x)=h(x)-g(2 x)+f(x)$ has a zero between 139 and 140 and is increasing for $x \geqslant 139$. Using (RS 1), (RS 2) and (RS 3), we obtain

$$
\varphi(n)>\pi(2 n)-\pi(n) \quad \text { whenever } n>139, n \neq n_{0} .
$$

For $12<n \leqslant 139$ and $n=n_{0}$ inequality (2) is verified numerically. Hence proposition (A) is true.

Denote by $\omega(n)$ the number of different prime factors of $n$. Proposition (B) is true, if the inequality

$$
\pi(n)-\omega(n)>\pi(2 n)-\pi(n)
$$

can be shown for $n>n_{1}$, and if (B) can be verified directly for $12<n \leqslant n_{1}$.**

Inequality (3) holds for all sufficiently large $n$. This follows easily from the trivial estimate

$$
\omega(n) \leqslant \frac{\log n}{\log 2}
$$

and Landau's result (see [1])

$$
2 \pi(x)-\pi(2 x)=2 \log 2 \cdot \frac{x}{\log ^{2} x}+o\left(\frac{x}{\log ^{2} x}\right) .
$$

Denote by $\vartheta(x)$ the logarithm of the product of all primes not greater than $x$. Connections between $\vartheta(x)$ and $\pi(x)$ are established by partial summation, for example

$$
\pi(x)=\frac{\vartheta(x)}{\log x}+\int_{2}^{x} \frac{\vartheta(u)}{u \log ^{2} u} d u .
$$

Rosser and Schoenfeld [3, Theorem 8$]$ proved the inequality

$$
|\vartheta(x)-x|<8.6853 \cdot \frac{x}{\log ^{2} x} \text { for } x>1 .
$$

From (5) and (RS 4) we deduce

$$
\left|\pi(x)-\frac{x}{\log x}-\frac{x}{\log ^{2} x}-\frac{2 x}{\log ^{3} x}\right|<9.5 \cdot \frac{x}{\log ^{3} x} \text { for } x \geqslant 10^{10}
$$

*C denotes Euler's constant.

** We prove (3) for $n>58$. 
by the same argument as in [2, Section 7]. *** Inequality (6) combined with (4) easily establishes the truth of (3) for $n \geqslant 10^{11}$.

Now we use (5.1) and (5.2) from [3] to get

$$
|\vartheta(x)-x|<0.001316 x \text { for } x \geqslant 10^{7}
$$

hence

$$
|\vartheta(x)-x|<0.85 \cdot \frac{x}{\log ^{2} x} \text { for } 10^{7} \leqslant x \leqslant 10^{11}
$$

In the same way as before we obtain

$$
\left|\pi(x)-\frac{x}{\log x}-\frac{x}{\log ^{2} x}-\frac{2 x}{\log ^{3} x}\right|<2 \cdot \frac{x}{\log ^{3} x} \text { for } 10^{7} \leqslant x \leqslant 10^{11} .
$$

This inequality enables us to deduce $(3)$ for $10^{7} \leqslant n \leqslant 10^{11}$.

In order to handle the interval $n \leqslant 10^{7}$ we use (4.1) and (4.2) from [2], giving

$$
\operatorname{li} x-\operatorname{li} x^{1 / 2}<\pi(x)<\operatorname{li} x \text { for } 11 \leqslant x \leqslant 10^{8} \text {. }
$$

The function

$$
r(x):=2\left(\operatorname{li} x-\operatorname{li} x^{1 / 2}\right)-\operatorname{li} 2 x
$$

is increasing when $x \geqslant 40$, and $r(x)-\log x / \log 2$ is increasing for $x \geqslant 2310$. Since $r(2310)>35>(1 / \log 2), \log 2310$, inequality (3) is true for $2310 \leqslant n \leqslant 10^{8}$. Since $\omega(n) \leqslant 4$ when $n<2310$ and $r(500)>4$, inequality (3) is true for $500 \leqslant n<$ 2310. Numerical calculations give the truth of (3) for $58<n<500$. In the interval $12<n \leqslant 58$ proposition (B) is verified directly. We remark that our calculations prove $\pi(2 x)<2 \pi(x)$ for $x \geqslant 11$. This result was announced by Rosser and Schoenfeld in the introduction to [3]. We further remark that the constants and the ranges of our estimates are not the best possible that can be obtained from the estimates of Rosser and Schoenfeld.

Finally, Santos asks whether there is a largest integer $n$ with the property

$\left(\mathrm{P}_{3}\right) \quad 1 \leqslant m \leqslant n$, g.c.d. $(n, m)=1$ and $m$ composite $\Rightarrow(n+m$ is prime $)$.

Since the inequality

$$
\varphi(n)-\pi(n)+\omega(n)>0
$$

is true for $n>281, \dagger$ it is possible to show that $n=12$ is the largest integer having property $\left(\mathrm{P}_{3}\right)$.

\footnotetext{
*** The values of the logarithmic integral li(x) needed for the proof can be calculated by using the power series expansion of the exponential integral $\operatorname{Ei}(x)$ and the relation $\operatorname{li}(x)=\operatorname{Ei}(\log x)$.

†This follows from (RS 1) and (RS 3).
} 
Institut für Mathematik Montanuniversität Leoben A-8700 Leoben, Austria

Fachbereich Mathematik J. W. Goethe-Universität Robert-Mayer-Strasse 10

D 6000 Frankfurt am Main

1. E. LANDAU, Handbuch der Lehre von der Verteilung der Primzahlen, Teubner, Leipzig und Berlin, 1909.

2. J. B. ROSSER \& L. SCHOENFELD, "Approximate formulas for some functions of prime numbers," Illinois J. Math., v. 6, 1962, pp. 64-94.

3. J. B. ROSSER \& L. SCHOENFELD, Sharper Bounds for the Chebyshev Functions $\theta(x)$ and $\psi(x)$, University of Wisconsin MRC Technical Summary Report \#1475, 1974.

4. B. R. SANTOS, "Twelve and its totitives," Math. Mag., v. 49, 1976, pp. 239-240. 